\title{
Speed Control with Low Armature Loss for Very Small Sensorless Brushed DC Motors
}

\author{
Jonathan Scott, Senior Member, IEEE, John McLeish, and Howell Round, Senior Member, IEEE
}

\begin{abstract}
A method for speed control of Brushed DC (BDC) motors is presented. It is particularly applicable to motors with armatures of less than one cubic centimetre. Motors with very small armatures are difficult to control using the usual PulseWidth Modulation (PWM) approach, and are apt to overheat if so driven. The technique regulates speed via the back EMF but does not require current-discontinuous drive. Armature heating in small motors under PWM drive is explained and quantified. The method is verified through simulation and measurement. Control is improved and armature losses are minimised. The method can expect to find application in miniature mechatronic equipment.
\end{abstract}

Index Terms-DC motor drives, Micromotors, Permanent magnet motors, Pulse width modulation, Variable speed drives, Rotating machine stability

\section{INTRODUCTION}

D C motors in speed-controlled applications have long been driven by means of a Pulse-Width Modulated (PWM) signal and chopper electronics [1]. Low-cost and mechanically-small brushed motors do not have a dedicated shaft sensor so back-EMF sensing is the established way to sense speed [2]. With PWM, speed sensing is achieved by running the motor in discontinuous-conduction mode and directly sampling the back-EMF that appears on the terminals after inductive flyback currents subside. It is possible to estimate speed by more complicated means, but this is not common [2], [3]. The increasing use of speed control with small motors by the discontinuous-conduction, back-EMF method is evidenced by the availability of application-specific microcontrollers intended for embedded application [4]. These low-cost ICs are provided with all the circuitry for analog sensing and PWM drive via H-bridge.

Problems arise when this method is applied to very small motors. The plant contains two real poles, and the speedsensor adds a zero-order hold. One pole is chiefly defined by the rotating mass of the system; this pole is typically the dominant one, and can vary with mechanical load. The second is associated with the motor inductance. The hold arises because the speed is sensed only once per period of the PWM drive, when the back-EMF is exposed during the off part of the drive cycle, after the inductive freewheel period. Because the drive must be current-discontinuous, the motor inductance limits the maximum PWM frequency, and so also the rate at which the speed is sampled. This rate, the PWM

The authors are with the Department of Electronic Engineering, the University of Waikato, Hamilton, New Zealand. e-mail: jonathanscott@ieee.org

Copyright (c) 2007 IEEE. Personal use of this material is permitted. However, permission to use this material for any other purposes must be obtained from the IEEE by sending a request to pubs-permissions@ieee.org..

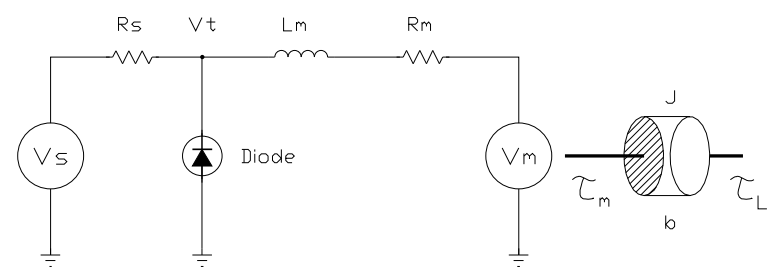

Fig. 1. Equivalent circuit of a small motor driven by a dc power supply. The motor terminal voltage, $V_{t}$, is the only node in the electrical circuit that can be measured directly. The motor inductance, series resistance, and back-EMF are designated $L_{m}, R_{m}$, and $V_{m}$ respectively. The flywheel diode need be considered only in the case of large-signal, pulsed operation.

frequency, is typically $50-400 \mathrm{~Hz}$. In the case of very small motors the mechanical pole and the PWM frequency lie close together. In a typical manufacturing situation speed control is achieved with a P- or PI-loop [5]. The coincidence of pole and sample frequency severely limits the performance of such a control strategy.

Operation in current-discontinuous mode leads to a high form factor in the armature current [8]. High form factor exacerbates loss in the armature. Very small motors tend to run with high power-density, and in low-cost designs there is often no provision for convection cooling. This can lead to heating in the motor.

The strategy proposed in section II addresses both the control and thermal issues. Section III quantifies the difficulty of controlling very small motors, and anticipates the measured results presented in section IV. Two motors have been used as examples throughout. Motor 1 is a low-cost cellphone vibrator motor of closed-can design with an armature of approximately $0.04 \mathrm{~cm}^{3}$. Motor 2 is a high-quality motor with an armature of approximately $0.3 \mathrm{~cm}^{3}$ designed to use shaft-driven convective cooling, unlike motor 1 that is in a sealed can. As appropriate, one or other motor or a comparison between the two will be used in the manuscript.

\section{Negative-Resistance Control}

Figure 1 shows the equivalent circuit representing a small dc motor driven by a dc power supply. The motor inductance, series resistance, and back-EMF are designated $L_{m}, R_{m}$, and $V_{m}$ respectively. The supply is represented as a voltage source and series resistance, $V_{S}$ and $R_{S}$. For a permanent magnet (PM) dc motor

$$
V_{m}=k_{e} s \Theta
$$

and

$$
T_{m}=k_{t} I_{m}
$$


where $\Theta$ is the shaft angular position and $s \Theta$ the angular velocity, $T_{m}$ is the shaft torque, and $k_{e}$ and $k_{t}$ are constant parameters of the motor. The aim of a speed controller is to keep angular velocity, and thus $V_{m}$, constant.

Working in Laplace transforms, Kirchoff's voltage law yields

$$
s \Theta=\frac{V_{S} k_{t}-\left(R_{S}+R_{m}+s L_{m}\right) T_{m}}{k_{e} k_{t}}
$$

where $T_{m}$ is the torque delivered to the armature from the electrical side; Newton's law yields

$$
T_{m}=s(J s+b) \Theta+T_{L}
$$

where $J$ is the mechanical moment of inertia at the armature shaft, $b$ is the damping ratio (frictional torque constant) of the system, and $T_{L}$ is any externally applied load torque. Combining 3 and 4 yields the shaft speed as a function of supply voltage in the open-loop case:

$$
s \Theta=\frac{k_{t} V_{S}-\left(R_{S}+R_{m}+s L_{m}\right) T_{L}}{k_{e} k_{t}+\left(R_{S}+R_{m}+s L_{m}\right)(J s+b)}
$$

A measure of the effectiveness of a controller is the change in speed that arises from a change in load torque:

$$
\frac{\partial s \Theta}{\partial T_{L}}=\frac{-\left(R_{S}+R_{m}+L_{m} s\right)}{k_{e} k_{t}+\left(R_{S}+R_{m}+L_{m} s\right)(J s+b)}
$$

When dealing with small motors, transients settle quickly, and so, provided the system is well-behaved, it is usually the steady-state response that is important. Let the steady-state change in speed with change in load be

$$
\mathcal{R}=\frac{\Delta \dot{\theta}}{\Delta \tau_{L}}=\frac{-\left(R_{S}+R_{m}\right)}{k_{e} k_{t}+b\left(R_{S}+R_{m}\right)}
$$

which will be small if $R_{S}+R_{m}$ is small. In motors of the size range found in small appliances $R_{m}$ and $R_{S}$ can be made sufficiently small that further speed regulation beyond the control of $V_{S}$ is not needed [9]. However, very small motors are becoming more common as mechatronic devices shrink, and they may have tens of ohms of series resistance.

The source resistance, $R_{S}$, can be set by electronics in the power supply. Putting

$$
R_{S}=-R_{m}
$$

then $\mathcal{R}$ goes to zero and steady-state error vanishes. Since a typical active dc power supply measures both its own output voltage, $V_{t}$, and its load current, $I_{m}$ in this case, it is straightforward to dial up a negative output resistance and achieve a desired steady-state back-EMF of, say $V_{\text {set }}$, by setting

$$
V_{t}=V_{\text {set }}+I_{m} R_{m}
$$

provided the system remains stable. Notionally this is equivalent to

$$
V_{t}=V_{S}-I_{m} R_{S}=V_{S}-I_{m}\left(-R_{m}\right)
$$

where $V_{S}$ is fixed, so one may refer to this strategy as "negative-resistance speed control". Although developed differently, this is very similar to the scheme reported in [2]. The difficulty with this method lies in the sensitivity of the regulation to errors in the estimate of $R_{m}$.

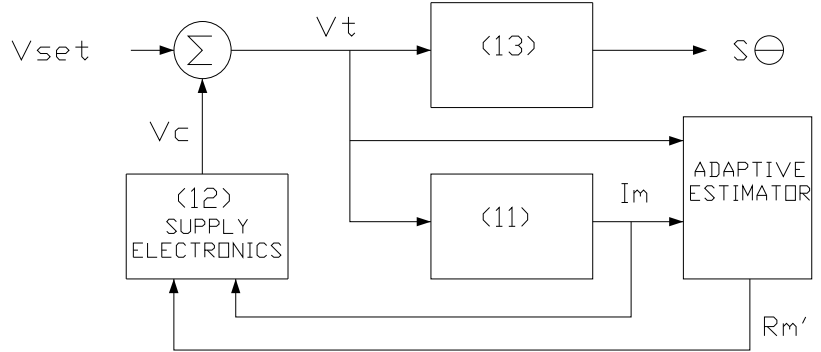

Fig. 2. Block diagram of a practical implementation of the controller. In the diagram, $V_{t}$ shows the motor terminal voltage and $s \Theta$ is the shaft speed we seek to control. The estimate of motor resistance is $R_{m}^{\prime}$. Numbers designate the corresponding equation from the text that describes the response of each block.

\section{A. Practical Implementation}

Figure 2 is the block diagram of a practical implementation of a controller. Returning to equations (1)-(4) but now solving for the armature current $I_{m}\left(V_{t}\right)$ yields

$$
I_{m}=\frac{V_{t}(J s+b)+k_{e} T_{L}}{k_{e} k_{t}+\left(R_{m}+s L_{m}\right)(J s+b)}
$$

and if the estimate of $R_{m}$ is designated as $R_{m}^{\prime}$, and the negative-resistance generator response is dominated by a single pole, $V_{c}\left(I_{m}\right)$ will be:

$$
V_{c}=\frac{-R_{m}^{\prime}}{1+\frac{s}{p}} I_{m}=\frac{-p R_{m}^{\prime}}{p+s} I_{m}
$$

while it is easy to show that the motor speed as a function of terminal voltage, $s \Theta\left(V_{t}\right)$, is

$$
s \Theta=\frac{k_{t} V_{t}-\left(R_{m}+s L_{m}\right) T_{L}}{k_{e} k_{t}+\left(R_{m}+s L_{m}\right)(J s+b)}
$$

In the case where $R_{m}^{\prime}=R_{m}$ and $p$ is large the steadystate conditions reduce to the situation of equation (9) and the steady-state error will vanish. In practice, performance will be limited by a pessimistic estimate of $R_{m}^{\prime}$ maintained in the interest of stability in the face of drift and noise.

\section{B. Controller Stability}

The control loop of figure 2 has the characteristic equation

$$
\begin{aligned}
0= & \frac{J L_{m}}{p} s^{3} \\
& +\left(\frac{b L_{m}}{p}+\frac{J R_{m}}{p}+J L_{m}\right) s^{2} \\
& +\left(\frac{R_{m} b}{p}+\frac{k_{e} k_{t}}{p}-J R_{m}^{\prime}+J R_{m}+b L_{m}\right) s \\
& +b R_{m}+k_{e} k_{t}-b R_{m}^{\prime}
\end{aligned}
$$

a cubic in canonical form $A s^{3}+B s^{2}+C s+D=0$. All roots of (14) will lie in the left half-plane provided all the coefficients are positive. Trivially $A>0$ and $B>0$, while $C>0$ if

$$
-R_{m}^{\prime}>-\left(R_{m}+\left[\frac{b L_{m}}{J}+\frac{R_{m} b+k_{e} k_{t}}{J p}\right]\right)
$$


and $D>0$ if

$$
-R_{m}^{\prime}>-\left(R_{m}+\frac{k_{e} k_{t}}{b}\right)
$$

In the desirable case of $p \rightarrow \infty$, equation (14) becomes equivalent to the denominator of equation (5), the former involving $R_{m}^{\prime}$ and the latter $R_{S}$. In practice, modern power electronics can make $p$ negligibly large, and it is equally effective to examine the denominator of equation (5) to assess stability. Some algebra leads to the following stability criterion:

$$
R_{S}>-\left(R_{m}+\frac{b L_{m}}{J}\right)
$$

provided $b L_{m} \ll J R_{m}$, readily satisfied for small motors.

Either of the above anaylses indicates that the system will become unstable should $R_{S}$ become a little larger in magnitude than $R_{m}$ and negative in sign, corresponding to the estimate $R_{m}^{\prime}$ being too large. This value below which $R_{S}$ cannot stray without instability, $R_{m}+b \frac{L_{m}}{J}$, can be thought of as the sum of two resistances, the first representing the electrical loss, and the second representing the mechanical loss transformed by the reactive components of the system, and so makes good intuitive sense.

\section{Adaptive Tuning}

If the estimate of $R_{m}$ given to the negative-resistance controller is too large it will set $R_{S}<-R_{m}-\frac{b L_{m}}{J}$ and the controller will become unstable. If it is too low, the steadystate speed error appears [3]. The solution is to measure $R_{m}$ dynamically and tune the controller. Formally, explicit SelfTuning Control (STC) through an Externally Excited Adaptive System (EEAS) is implemented [10]. In practice this amounts to occasionally estimating $R_{m}$ by introducing a small perturbation in $V_{S}$ at a frequency too high to affect the mechanical operation, while measuring the resulting changes in $V_{t}$ and $I_{m}$. If load torque is constant, the dynamic impedance can be written

$$
Z_{T}=\frac{\partial V_{t}}{\partial I_{m}}=\frac{k_{e} k_{t}}{(J s+b)}+\left(R_{m}+s L_{m}\right)
$$

If $J$ is sufficiently large and $L_{m}$ sufficiently small, there will be a frequency $s=\jmath \omega_{m}$ where an estimate of $R_{m}$ can be obtained by simple division since $Z_{T} \rightarrow R_{m}$. If these assumptions cannot be made, it becomes necessary to choose a value of $\omega_{m}$ where the electrical pole is not negligible and the computation of $R_{m}$ requires a phase measurement to resolve the real part of $Z_{T}$.

As pointed out in reference [10], the adaptive system method relies for its effectiveness upon the assumption that the system parameters, $R_{m}$ in this case, change much more slowly than the disturbances addressed by the inner control loop. This assumption is very comfortably made even in the case of very small motors.

\section{Simulation}

It was asserted in the introduction that feedback control was problematic in the case of very small motors. In this section this assertion will be demonstrated quantitatively by means

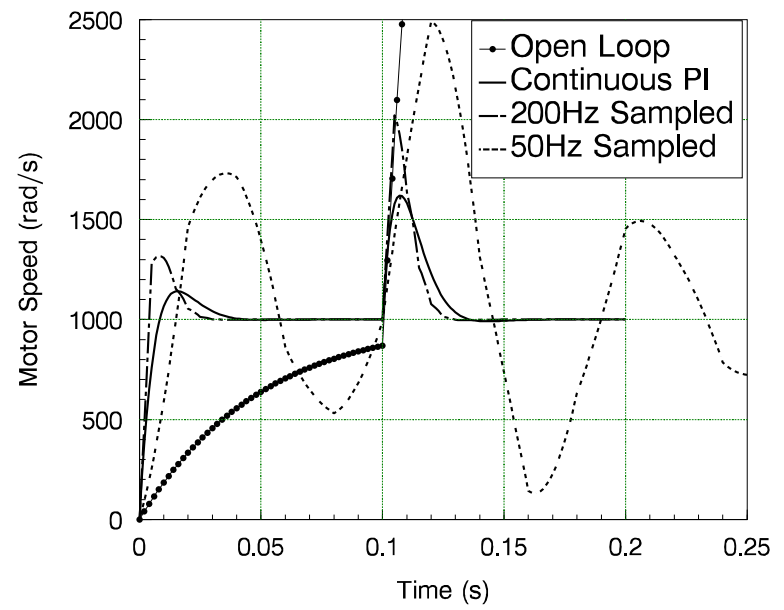

Fig. 3. Simulated speed-time curves for Motor 1. A step load disturbance occurs at $t=0.1 \mathrm{~s}$. The trace of connected dots shows the open loop speed as a function of time with sufficient voltage applied to achieve a steady-state speed of 1000 radians/second. The continuous line trace shows the motor response with tuned, continuous-time, PI feedback control. The dash-dot trace shows the motor response with tuned PI feedback control but in the presence of a zero-order hold at $200 \mathrm{~Hz}$. The discrete-dot trace shows the motor response with retuned PI feedback control in the presence of a zero-order hold at $50 \mathrm{~Hz}$.

\begin{tabular}{|c|c|c|c|c|c|}
\hline & $R_{m}(\Omega)$ & $L_{m}(\mathrm{mH})$ & $k_{e}(\mathrm{Nm} / \mathrm{A})$ & $\mathrm{J}\left(\mathrm{kgm}^{2} / \mathrm{s}^{2}\right)$ & $\mathrm{b}(\mathrm{Nms})$ \\
\hline motor 1 & 14 & 0.03 & 0.00034 & $1.2 \times 10^{-9}$ & $1.5 \times 10^{-8}$ \\
\hline motor 2 & 52 & 6.8 & 0.001 & $3.6 \times 10^{-9}$ & $1 \times 10^{-7}$ \\
\hline
\end{tabular}

TABLE I

PARAMETERS OF THE MOTORS USED IN SIMULATION.

of simulation. Figure 3 shows a number of speed-time traces simulated for motor 1 , pictured in figure 4 . Initially, the openloop response of the motor driven by a constant voltage is simulated. Then the same motor is simulated when controlled by a Proportional-plus-Integral (PI) loop with continuous speed sensing. Finally the simulation is extended with the addition of zero-order hold functions at $200 \mathrm{~Hz}$ and $50 \mathrm{~Hz}$ to represent the effect of back-EMF speed sampling. When unloaded this motor has a mechanical pole around 20 radians/second, and an electrical pole around $4.6 \times 10^{5}$ radians/second. The simulations were carried out in VisSim [6]. The parameters of the test motors are given in table I. The value of $k_{e}$ is assumed equal to $k_{t}$, as torque cannot be directly measured.

It is clear from the data in figure 3 that a continuous-time, proportional-integral feedback controller improves the speed regulation of the motor in comparison to open-loop operation with a fixed supply voltage, as expected. The motor comes up to speed and handles a test disturbance, settling in less than $50 \mathrm{~ms}$, with zero constant error. The open-loop case shows a recovery time constant of more than $50 \mathrm{~ms}$ and massive (offscale) error in response to the same disturbance. However, when a zero-order hold appears the situation changes. In the case of a $200 \mathrm{~Hz}$ PWM frequency there is a clear shift towards instability. In the case of a $50 \mathrm{~Hz}$ PWM frequency, the original controller goes completely unstable. The oscillatory trace shown in the plot is a barely-stable result that might be 


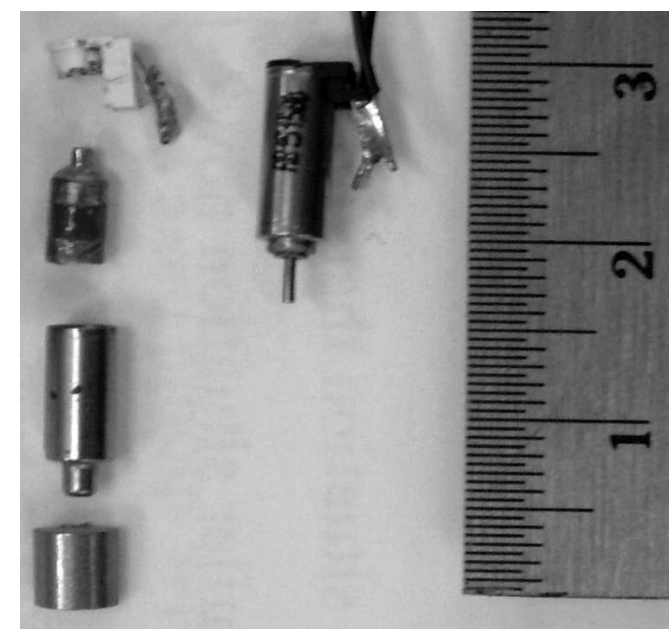

Fig. 4. Picture of motor 1 to represent the size range of concern in this work. The ruler shows centimetres. Motor 1 is shown both whole and dismantled to expose the armature. This is the motor whose parameters are used in the simulations appearing in figure 3 and that is used for the measurements presented in figure 6 .

called an optimum result. ${ }^{1}$ It settles to the desired speed, but not for many hundreds of milliseconds, and there is excessive overshoot.

The control strategy of section II is proposed to address the problems that arise with sampling speed between PWM drive pulses. It effectively removes the zero-order hold from the speed-control plant.

In practice, small motors used with back-EMF speed sensing are often controlled using proportional-only feedback for reasons of economy and simplicity of tuning, given the highly variable mecanical load associated with the dominant pole. In section IV the performance of several proportionalonly controllers will be compared with reference to motor 2 . Motor 2 was selected because a number of commercial and enthusiast controller designs are available for use with this size and type of motor, and these are included in the comparison. Figure 5 shows simulated results for two cases: Openloop, voltage-driven operation in response to a typical step disturbance, and the response to the same disturbance in the presence of proportional-only feedback control, using voltageswitched, PWM drive and with $50 \mathrm{~Hz}$ speed sampling, using the parameters of motor 2 .

The disturbance produces a 33\% change in speed in the open loop case. With proportional feedback, this can be reduced to approximately $14 \%$, but only with the gain constant $K_{P}$ so high as to be close to instability, as indicated by the overshoot in the response. If continuous control is applied to the same motor, even proportional-only feedback can produce a nearperfect result (less than $0.5 \%$ error and millisecond settling time), because the motor's electrical pole is so far from the mechanical pole. This simple simulation highlights both the difficulty presented by the back-EMF sensing approach, and the opportunity for improvement.

\footnotetext{
${ }^{1}$ Precisely what constitutes an optimum result is open to debate. There is a trade-off between settling time and intensity of overshoot. No PI controller can produce a pleasing result. This is a representative result selected to exemplify the difficulty.
}

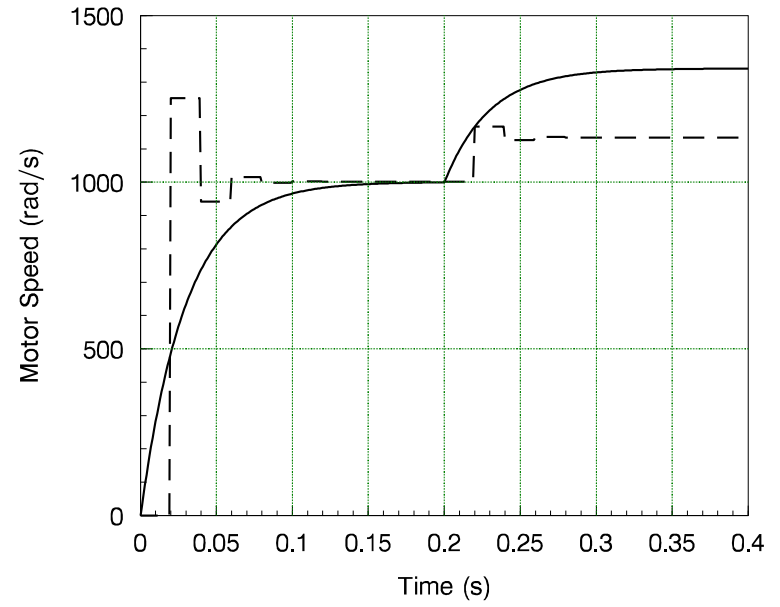

Fig. 5. Simulated speed-time curves for Motor 2. A step load disturbance occurs at time $t=0.2 \mathrm{~s}$. The solid trace shows speed in the open-loop case. The dash-dot trace shows the motor response with proportional control but in the presence of a zero-order hold at $50 \mathrm{~Hz}$.

\section{Measured Results}

\section{A. Motor Heating}

In the introduction it was stated that one of the objections to current-discontinuous PWM in the case of very small motors was excess heating in the motor. This condition is discussed anecdotally, and there is active research into the effect of PWM on motor laminations [7]. However, the authors could find no discussion of a heating problem associated with small motors in the professional literature. Therefore this assertion will first be demonstrated quantitatively.

In the equivalent model of figure 1 dissipation in the armature of the motor is represented by the power dissipated in $R_{m}$. In the case of pure dc drive, the armature dissipates a power $P_{A}=R_{m} I_{m}^{2}$ when consuming an electrical power of $P_{e}=P_{M}+P_{A}=V_{t} I_{m}$ and delivering a mechanical power $P_{M}=V_{m} I_{m}$.

For back-EMF sensing, operation must be current discontinuous, and the motor terminals open-circuit after the inductive flywheel period. The PWM drive is represented by the source switching between a fixed voltage level and an open circuit, not by the source taking on a square-wave shape as might be assumed at first. The purpose of the diode in figure 1 is to provide a low source impedance during inductive freewheeling. Pulsing is represented by $R_{S}$ taking on alternately a small value and a value of $\infty$ with some frequency $f_{\mathrm{PWM}}$ and duty cycle $0 \leq \mathcal{D}<1$. The armature loss in the case of PWM drive when delivering the same mechanical power is found to be [8]:

$$
P_{A_{\mathrm{PWM}}}=\frac{P_{A}}{\mathcal{D}}
$$

Figure 6 shows measurements made of case temperature as a function of time on motor 1 . In both cases the motor is operated under the same mechanical conditions, but with puredc drive in one case and PWM in the other case. Asymptotic temperature rise is inversely proportional to duty cycle, as predicted by equation (19). 


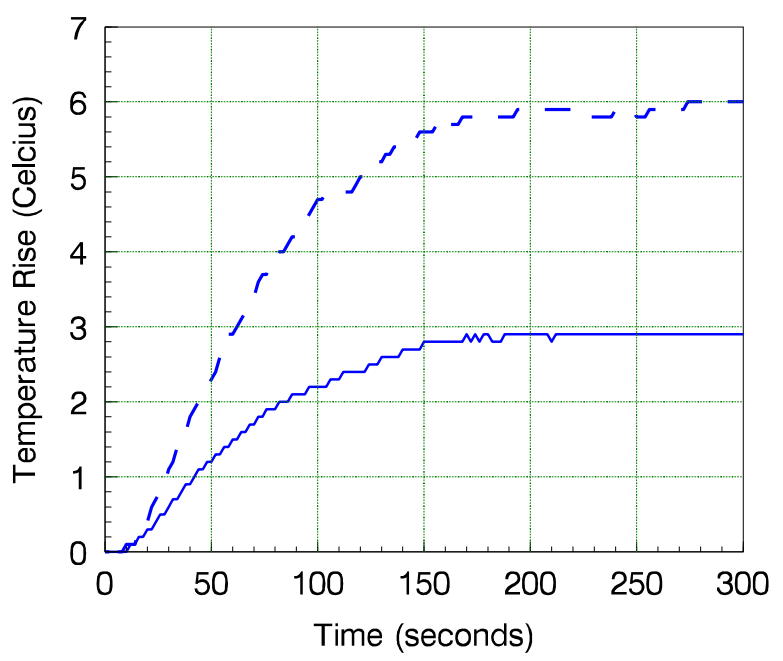

Fig. 6. Case temperature rise for Motor 1 at 6000 RPM using DC and $50 \%$ duty-cycle pulsed drive. The dashed trace identifies the pulsed drive. Temperature rise corresponds exactly with the armature current form factor as predicted.

Figure 7 shows measurements made of case temperature as a function of time on motor 2. This motor has shaft-driven convective cooling, unlike motor 1 that is in a sealed can. In this experiment the drive voltage and PWM frequency are kept constant, and the speed is varied by means of the PWM duty cycle. The results show that the temperature rise increases with decreasing duty cycle, speed, and power. This is attributed to the increased form factor of the armature current and reduced cooling effect accompanying reduced shaft speed overcoming the reduced power being delivered. Other effects can exacerbate the problem. For example, the armature resistance of motor 2 increases with frequency, as shown in figure 8 . Since pulsed drive delivers significant energy at frequencies that are harmonics of the PWM frequency, the increased $R_{m}$ results in greater loss at the harmonic frequencies.

\section{B. Speed Regulation}

In order to demonstrate the viability of this approach, both a fixed negative- $\mathrm{R}$ controller and an elementary adaptive version were implemented. These were compared with four alternatives, namely plain constant-voltage drive, two commerciallyavailble, EMF-sensing, proportional-only PWM feedback controllers, and an EMF-sensing controller that can implement PID control. The negative-R controller implemented only proportional control in this test, so that a comparison of likeagainst-like is obtained. ${ }^{2}$

It is customary to test a motor with a mechanical arrangement that can apply a known constant load torque, such as a disk brake with the caliper applying force to a scale. This arrangement is impractical in the case of very small motors. Additionally, friction losses dominate any mechanical system on this scale, so that only relative rather than absolute

\footnotetext{
${ }^{2}$ The reader will appreciate that the advantage of the negative- $\mathrm{R}$ approach is expected to be greater with the addition of an integral component of feedback, but the comparison becomes much more complicated and is beyond the scope of this manuscript.
}

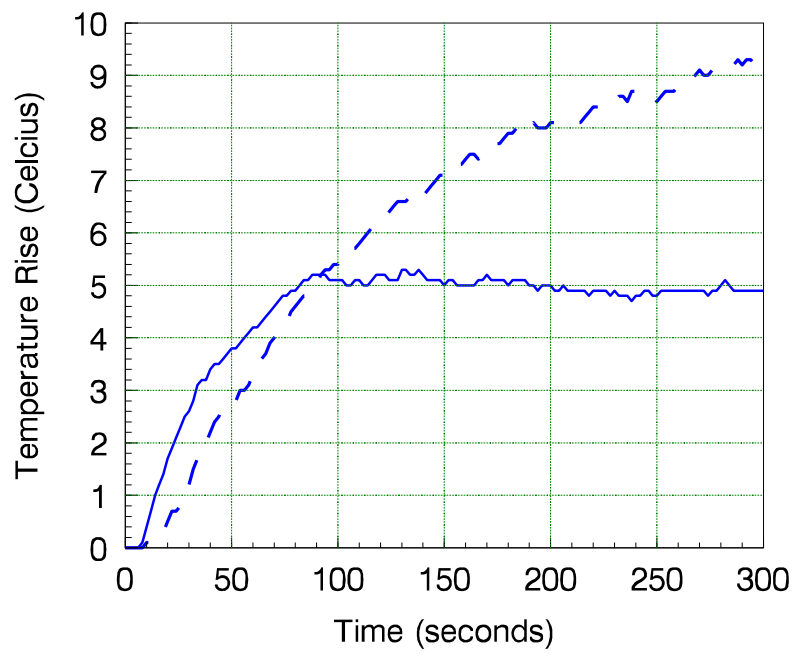

Fig. 7. Case temperature rise for Motor 2 with dc and $25 \%$ duty-cycle pulsed drive. The source voltage remained constant, whether pulsed or dc. The pulse drive frequency was $490 \mathrm{~Hz}$. Load was kept constant and speed alowed to vary. Motor 2 uses forced-convection cooling.

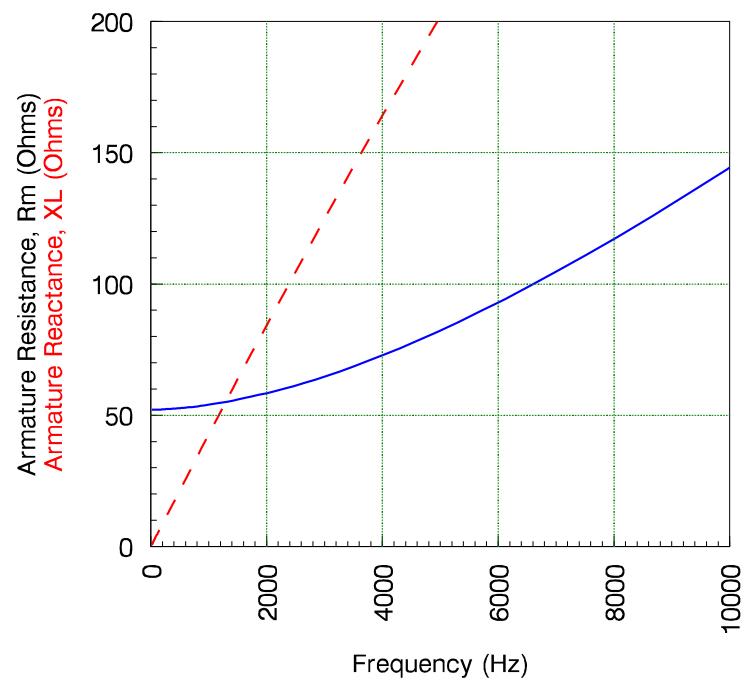

Fig. 8. The armature resistance of motor 2 plotted as a function of frequency. The measurement was made with the motor stationary using an HP4192 vector impedance meter. The inductive reactance component is plotted in the dashed line for comparison.

comparisons are practical. Instead a mechanical system is used to apply a fixed load using a weight via reduction gears. The weight could be alternately raised and lowered with the same set speed. The test consisted of having the motor alternately raise and lower the weight in a 15-second cycle, and reporting the difference in time taken over the raising and lowering phases of the test cycle. There has been no attempt to characterise the loading of this system in detail, but it is kept constant throughout the tests.

This mechanical setup is used to assess the regulation, $\mathcal{R}$, of the various controllers, on motor 2 . Figure 9 shows the results. The "PWM with PID" controller and the negative resistance controllers with and without adaptive adjustment of the estimate of armature resistance were designed by the authors, the others were purchased [12], [13]. The negative resistance 


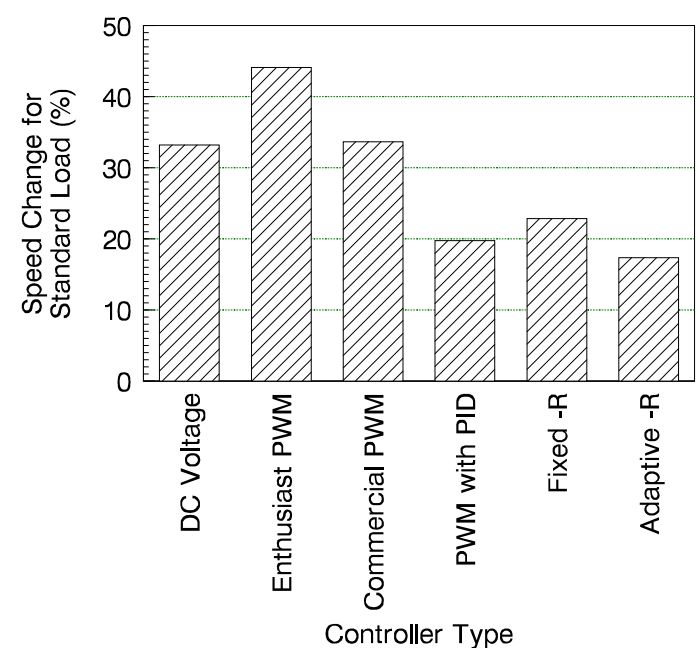

Fig. 9. Performance of various controller methods applied to motor 2, a high-quality PM BDC motor with an armature of approximately $0.3 \mathrm{~cm}^{3}$. The adjustable controllers are set up for stable operation with the motor unloaded. All controllers were then tested with the same load.

controllers implemented only proportional $(\mathrm{P})$ feedback. The PID controller had a $125 \mathrm{~Hz}$ PWM frequency, while the design of [12] used $200 \mathrm{~Hz}$ and and the design of [13] used $50 \mathrm{~Hz}$. The PID controller is tuned to give modest overshoot and so has a long settling time. The 15-second measurement duration explains why it yields a speed error.

It is clear that in the case of this small motor even the commercial controller is worse than a well-regulated dc supply. To an extent this is not surprising for two reasons: The purchased controllers did not allow loop gain to be changed but were factory preset, and they necessarily present a high impedance for a part of the cycle to expose the back-EMF as noted earlier. ${ }^{3}$ The controller described in reference [13] was on the edge of stability with an unloaded motor so it was possible to conclude that it had a good choice of loop gain given the constraint of unconditional stability. The PID controller shows a small advantage over the proportional-only designs, but this advantage is slight, on account of the long settling times that result.

The fixed negative-resistance design is limited by the precision of its estimate of the real series resistance. Of course, results could be arbitrarily improved, at the expense of its robustness. Rather than tweak the estimate to achieve a superb result for publication as was done in reference [2], a result that corresponds to a value chosen to give stable operation in spite of varying connection resistances and drifts is given. The results shown correspond to a very pessimistic estimate. A similarly-pessimistic position is taken when implementing the adaptive estimation of the resistance. The controller will not falter even as the series resistance is varied by several ohms in the space of seconds, or in the presence of commutator noise. It is reasonable to expect that refining the adaption algorithm will allow robust "continuous" PI control.

\footnotetext{
${ }^{3}$ It should be noted that these controllers aquire an increasing advantage as the connection resistance between the source and the motor increases, as would be the case with long wires.
}

\section{CONCLUSION}

It has been shown that speed control using currentdiscontinuous PWM is limited and can be quite ineffective in the case of motors with small armatures, meaning less than approximately 1 cubic centimetre in volume. PWM also increases armature resistive losses, leading to significant heating especially at low speeds. This problem has been explained and quantized. It is evident that a number of commercial designs purporting to provide superior performance through feedback control are no better than a constant-voltage, lowimpedance supply. An adaptive, negative-resistance strategy for speed regulation by means of back-EMF sensing has been presented. The theory is backed up by simulated and measured results. The superiority of the negative-resistance method over alternatives when applying proportional control has been shown. This should extend to PI control as required. It is to be expected that adaptive, negative-resistance speed control will find application with the growing number of small mechatronic devices.

\section{REFERENCES}

[1] H.F. Weber, "Pulse-Width Modulation DC Motor Control", IEEE Transactions on Industrial Electronics and Control Instrumentation, Volume IECI-12, Issue 1, March 1965, pp:24-28.

[2] S. Rajaram and S. Murugesan, "A New Method for Speed Measurement/Control of DC Motors", IEEE Transactions on Instrumentation and Measurement, Volume 27, Issue 1, March 1978 pp99-102.

[3] P. Chevrel and S. Siala, "Robust DC-Motor Speed Control Without any Mechanical sensor", Proceedings of the 1997 IEEE International Conference on Control Applications, Hartford, CT, October 5-7, 1997, pp244-246.

[4] Microchip Technology Inc., "Motor Control Devices", [Online]. Available: http://www.microchip.com/ParamChartSearch/chart.aspx? branchID $=55 \&$ mid $=10$, retrieved January 2008 .

[5] H. Wertz and F. Schutte, "Self-tuning speed control for servo drives with imperfect mechanical load", Conference Record of the 2000 IEEE Industry Applications Conference, Volume 3, 2000, pp:1497-1504.

[6] Visual Solutions Incorporated, VisSim embedded system development software, [Online]. Available: http://vissim.com/. retrieved January 2008.

[7] Z. Gmyrek, A. Boglietti, and A. Cavagnino, "Iron Loss Prediction With PWM Supply Using Low- and High-Frequency Measurements: Analysis and Results Comparison", IEEE Transactions on Industrial Electronics, vol. 55, no. 4, April 2008, pp1722-1728.

[8] N Mohan, T M Undeland, and W P Robbins, Power Electronics, John Wiley and Sons, 3rd Edition, 2003.

[9] Thierry Castagnet and Jean Nicolai, "Digital Control for Brush DC Motor", IEEE TRANSACTIONS ON INDUSTRY APPLICATIONS, vol. 30, no. 4, July/August 1994, pp883-888.

[10] Astrom, K.J., "Adaptive feedback control", Proceedings of the IEEE, Volume 75, Issue 2, Feb. 1987, pp:185-217.

[11] K. Ohnishi, M. Matsui, and Y. Hori, "Estimation, identification, and sensorless control in motion control system", Proceedings of the IEEE, Volume 82, Issue 8, Aug 1994, pp:1253-1265.

[12] J. Clarke, and L. Simpson, "L'il pulser train controller" Silicon Chip Magazine, 5 Feb. 2001.

[13] Hornby International Model Railways, HM2000 R8012 Product description, [Online]. Available: http://www.hornby.com/ power-and-accessories-90/hm-2000-r8012/, retrieved February, 2008. 


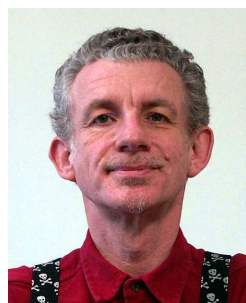

Jonathan Scott (S'82-M'97-SM'98) was born in Brisbane, Australia in 1956. He received the B.Sc., B.E., M.Eng.Sc., and Ph.D. degrees from The University of Sydney, Sydney, Australia, in 1977, 1979, 1983 and 1997 respectively.

He is currently the Foundation Professor of Electronics at the University of Waikato, Hamilton, New Zealand. From 1998 to 2006, he was with the Microwave Technology Center, Hewlett-Packard (now Agilent Technologies), Santa Rosa, CA, where he was responsible for advanced measurement systems. In 1997 and 1998, he was Chief Engineer with RF Technology, Sydney, N.S.W., Australia. Prior to 1997, he was with the Department of Electrical Engineering, University of Sydney. He is a Professorial Fellow of Macquarie University. He has served on committees of the Standards Association of Australia and the NRC Review Panel for the National Institute of Standards and Technology (NIST). He has authored over 80 refereed publications, several book chapters, and a textbook. He holds nine patents.

Professor Scott's interests include RF and microwave systems, compound semiconductor devices, electronic circuit linearity and nonlinear measurement, sensors and instrumentation, power electronics, and mechatronics.

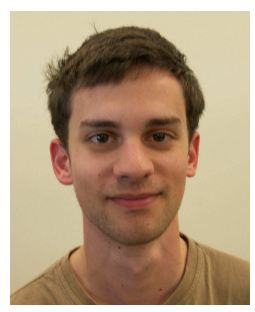

John McLeish expects to graduate at the end of 2008 with a Bachelors degree in Engineering from The University of Waikato.

He held a Summer Research Scholarship in 20072008 for study in Motor Control Systems for Small Scale Applications. His interests include mechatronics, optical systems and power electronics.

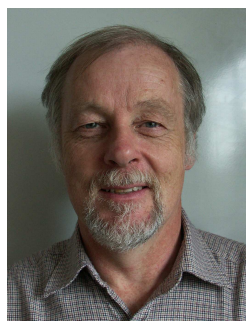

W. Howell Round (M'00-SM'01) received the $\mathrm{BSc}(\mathrm{Tech})$ degree in physics from the University of Waikato, New Zealand, the MSc degree in Medical Physics from the University of Surrey, UK, and the $\mathrm{PhD}$ in Electrical and Electronic Engineering from the University of Canterbury, New Zealand. After working as a clinical medical physicist in Australia and New Zealand, he joined the University of Waikato, NZ, in 1985 where he is a senior lecturer in physics and electronic engineering. His research interests are in electronics, biomedical engineering, inertial sensor navigation of motor vehicles, Monte Carlo modelling in medical imaging and radiation dosimetry, and medical physics workforce training, analysis and planning. He is a fellow of the New Zealand Institute of Physics and the Australasian College of Physical Scientists and Engineers in Medicine. 